\title{
Superconformal M2-branes and generalized Jordan triple systems
}

\author{
Bengt E W Nilsson ${ }^{1}$ and Jakob Palmkvist ${ }^{1,2}$ \\ ${ }^{1}$ Fundamental Physics, Chalmers University of Technology, SE-412 96 Göteborg, Sweden \\ 2 Max Planck Institute for Gravitational Physics, Albert Einstein Institute, Am Mühlenberg 1, \\ DE-14476 Golm, Germany \\ E-mail: tfebn@chalmers.se and jakob.palmkvist@aei.mpg.de
}

Received 13 January 2009, in final form 9 February 2009

Published 2 March 2009

Online at stacks.iop.org/CQG/26/075007

\begin{abstract}
Three-dimensional conformal theories with six supersymmetries and $S U(4) R$ symmetry describing stacks of M2-branes are here proposed to be related to generalized Jordan triple systems. Writing the four-index structure constants in an appropriate form, the Chern-Simons part of the action immediately suggests a connection to such triple systems. In contrast to the previously considered 3-algebras, the additional structure of a generalized Jordan triple system is associated with a graded Lie algebra, which corresponds to an extension of the gauge group. In this paper we show that the whole theory with six manifest supersymmetries can be naturally expressed in terms of such a graded Lie algebra. Also the Bagger, Lambert and Gustavsson theory with eight supersymmetries is included as a special case.
\end{abstract}

PACS numbers: $11.25 . \mathrm{Hf}, 11.25 . \mathrm{Yb}$

\section{Introduction}

A three-dimensional maximally $(\mathcal{N}=8)$ superconformal theory was recently constructed by Bagger, Lambert and Gustavsson (BLG) in [1-4]. The BLG theory was originally proposed to describe multiple M2-branes. An interesting aspect of this theory is that it contains a Chern-Simons term [5] making the BLG theory potentially interesting also for condensed matter applications. The multiple M2-brane interpretation has, however, met with a number of problems having to do with the algebraic structure on which the theory is based. The theory contains a kind of four-index structure constant for a 3-algebra with a Euclidean metric. This 3 -algebra has, however, been proven [6,7] to have basically only one realization, $\mathcal{A}_{4}$, related to the ordinary Lie algebra $s o(4)$ through its totally antisymmetric epsilon tensor. This limits the role of the BLG theory to stacks of two M2-branes $[8,9]$. 
By relaxing the assumption that the metric on the algebra should be positive definite [10] any Lie algebra can be accommodated. The drawback of using a degenerate metric as done in [10] is that it produces a set of field equations which cannot be integrated to a Lagrangian if the zero norm mode is not assumed constant. This subsequently led to a number of attempts to use a non-degenerate but Lorentzian metric [11-13]. Again there are problems; these theories make sense only provided the negative norm modes can be rendered harmless. Even when this is the case they are of real interest only if they contain genuine M2-physics instead of just providing a reformulation of the D2-brane. For some recent results in this direction, see [14-17].

From the work of [10] it was also clear that the structure constants need not be totally antisymmetric. This might be interesting since this property seems to be part of the reason why only one realization, related to $S O(4)$, of the fundamental identity can be constructed in the Euclidean case. In fact, as realized by Aharonyet al (ABJM) [18], by reducing the number of linearly realized supersymmetries from the maximal $\mathcal{N}=8$ to $\mathcal{N}=6$ this nogo theorem can be avoided. Following [19], the authors of [18] (see also [20, 21]) used a construction with the fields in the bi-fundamental representation of $U(N) \times U(N)$ and without any reference to the four-index structure constants. However, in a work following this Bagger and Lambert [22] pointed out that if reinstating the four-index structure constants there are interesting implications for their antisymmetry properties. In particular, six supersymmetries are compatible with structure constants which are not totally antisymmetric.

The purpose of this paper is to write the structure constants in yet another form which suggests the possibility of relating them to certain algebraic structures, known as generalized Jordan triple systems. Since this result will rely on embeddings into infinite-dimensional graded Lie algebras $g$ we should here mention that embeddings into finite-dimensional ones are also possible [23-26] but then $g$ is a (three graded) Lie superalgebra.

The paper is organized as follows. In section 2, we review the ABJM theory and present the Lagrangian in terms of four-index structure constants as described in [22]. In section 3, we then provide a reformulation of this theory in terms of structure constants adapted to triple systems. Some relevant aspects of generalized Jordan triple systems and the associated graded Lie algebra are summarized in section 4 . The last section contains conclusions and some further comments.

\section{The ABJM M2-theory}

The BLG theory contains three different fields; the two propagating ones $X^{I}{ }_{a}$ and $\Psi_{a}$, which are three-dimensional scalars and spinors, respectively, and the auxiliary gauge field $\tilde{A}_{\mu}{ }^{a} b$. Here the indices $a, b, \ldots$ are connected to the 3 -algebra and some $n$-dimensional basis $T^{a}$, while the $I, J, K, \ldots$ indices are $S O(8)$ vector indices. The spinors transform under a spinor representation of $S O(8)$ but the corresponding index is not written out explicitly. Indices $\mu, v, \ldots$ are vector indices on the flat M2-brane world volume.

Using these fields one can write down $\mathcal{N}=8$ supersymmetry transformation rules and covariant field equations. This is possible without introducing a metric on the 3-algebra. In such a situation the position of the indices on the structure constants is fixed as $f^{a b c}{ }_{d}$. The corresponding fundamental identity needed for supersymmetry and gauge invariance then reads [1-4],

$$
f_{g}^{a b c} f_{d}^{e f g}=3 f^{e f[a} f^{b c] g}
$$

which can be written in the following alternative but equivalent form [10]:

$$
f_{g}^{[a b c} f_{d}^{e] f g}=0 .
$$


The construction of a Lagrangian requires the introduction of a metric on the 3-algebra. As discussed above, if one wants to describe more general Lie algebras than $s o(4)$, this metric must be degenerate [10] or non-degenerate but indefinite [11-13]. Finally, to construct an action one also needs to introduce the basic gauge field $A_{\mu a b}{ }^{3}$ which is related to the previously defined gauge field and structure constants as follows:

$$
\tilde{A}_{\mu b}^{a}=A_{\mu c d} f_{b}^{c d a} .
$$

The BLG Lagrangian is [3]

$$
\begin{aligned}
\mathcal{L}=-\frac{1}{2}\left(D_{\mu} X^{I a}\right)\left(D^{\mu} X^{I}{ }_{a}\right)+\frac{\mathrm{i}}{2} \bar{\Psi}^{a} \gamma^{\mu} D_{\mu} \Psi_{a}+\frac{\mathrm{i}}{4} \bar{\Psi}_{b} \Gamma_{I J} X_{c}^{I} X^{J}{ }_{d} \Psi_{a} f^{a b c d} \\
-V+\frac{1}{2} \varepsilon^{\mu \nu \lambda}\left(f^{a b c d} A_{\mu a b} \partial_{\nu} A_{\lambda c d}+\frac{2}{3} f^{c d a}{ }_{g} f^{e f g b} A_{\mu a b} A_{\nu c d} A_{\lambda e f}\right)
\end{aligned}
$$

where the potential is given by

$$
V=\frac{1}{12} f^{a b c d} f^{e f g}{ }_{d} X^{I}{ }_{a} X^{J}{ }_{b} X^{K}{ }_{c} X^{I}{ }_{e} X^{J}{ }_{f} X^{K}{ }_{g} .
$$

Note that in terms of $\tilde{A}$ the Chern-Simons term becomes

$$
\mathcal{L}_{\mathrm{CS}}=\frac{1}{2} \varepsilon^{\mu \nu \lambda}\left(A_{\mu a b} \partial_{\nu} \tilde{A}_{\lambda}{ }^{a b}+\frac{2}{3} A_{\mu}{ }^{a}{ }_{b} \tilde{A}_{\nu}{ }^{b}{ }_{c} \tilde{A}_{\lambda}{ }^{c}{ }_{a}\right)
$$

and that the fundamental identity implies that, in the variation of the last term, the structure constants can be associated with any two of the three vector fields.

Following ABJM [18] we now rewrite this in a form which has only six manifest supersymmetries and manifest $S U(4) R$-symmetry. As emphasized by these authors, this is naturally done using matter fields in the bi-fundamental representation [19] of $U(N) \times U(N)$, and no reference to 3-algebras and their structure constants is needed. However, for the purpose of this paper we need to reinstate the four-index structure constants. Fortunately, this was discussed in detail in a recent work by Bagger and Lambert [22].

The ABJM action is expressed in terms of complex scalar fields $Z^{A}{ }_{a}$ and spinors $\Psi_{A a}$ with the capital indices transforming in fundamental and anti-fundamental representations of the $S U$ (4) $R$-symmetry, respectively. If rewritten in terms of four-index structure constants as done in [22] (but rescaled by a factor of 2), the ABJM action reads

$$
\begin{aligned}
\mathcal{L}=-\left(D_{\mu} Z^{A}{ }_{a}\right) & \left(D^{\mu} \bar{Z}_{A}{ }^{a}\right)-\mathrm{i} \bar{\Psi}^{A}{ }_{a} \Gamma^{\mu} D_{\mu} \Psi_{A}{ }^{a} \\
& -\mathrm{i} f^{a b c d} \bar{\Psi}^{A}{ }_{d} \Psi_{A a} Z^{B}{ }_{b} \bar{Z}_{B c}+2 \mathrm{i} f^{a b c d} \bar{\Psi}^{A}{ }_{d} \Psi_{B a} Z^{B}{ }_{b} \bar{Z}_{A c} \\
& -\frac{\mathrm{i}}{2} \epsilon_{A B C D} f^{a b c d} \bar{\Psi}^{A}{ }_{c} \Psi^{B}{ }_{d} Z^{C}{ }_{a} Z^{D}{ }_{b}-\frac{\mathrm{i}}{2} \epsilon^{A B C D} f^{c d a b} \bar{\Psi}_{A c} \Psi_{B d} \bar{Z}_{C a} \bar{Z}_{B d} \\
& -V+\frac{1}{2} \epsilon^{\mu \nu \lambda}\left(f^{a b c d} A_{\mu a b} \partial_{\nu} A_{\lambda c d}+\frac{2}{3} f^{c d a}{ }_{g} f^{e f g b} A_{\mu a b} A_{\nu c d} A_{\lambda e f}\right),
\end{aligned}
$$

where the potential can be written

$$
\begin{aligned}
& V=\frac{2}{3} \Upsilon^{C D}{ }_{B d} \bar{\Upsilon}_{C D}{ }^{B d}, \\
& \Upsilon^{C D}{ }_{B d}=f^{a b c}{ }_{d} Z^{C}{ }_{a} Z^{D}{ }_{b} \bar{Z}_{B c}+f^{a b c}{ }_{d} \delta^{[C}{ }_{B} Z^{D]}{ }_{a} Z^{E}{ }_{b} \bar{Z}_{E c} .
\end{aligned}
$$

In order to write this action one needs a metric on the 3-algebra to raise and lower 3-algebra indices. The structure constants appearing in this formulation of the $\mathcal{N}=6$ ABJM theory [22] are antisymmetric in the first pair of indices as well as in the second pair while complex conjugation is defined to interchange the two pairs of indices.

As we will see below the need for an explicit metric in the Lagrangian can be eliminated by writing the structure constants as $f^{a b}{ }_{c d}$ or $f^{a}{ }_{b{ }^{c}{ }_{d}}$ (which we will see later are in fact related to each other). This will also require the introduction of a graded Lie algebra in a way that will be explained in the following section.

3 However, already gauge invariance of the field equations requires this gauge field [10]. 


\section{Structure constants adapted to triple systems}

Our next goal is to try to relate the M2-brane to generalized Jordan triple systems. The first step is to rewrite the $\mathcal{N}=6 \mathrm{M} 2$-theory as formulated at the end of the previous section in terms of structure constants with two upper and two lower indices, which are antisymmetric in each pair separately,

$$
f^{a b}{ }_{c d}=f^{[a b]}{ }_{c d}=f^{a b}{ }_{[c d]} .
$$

The crucial difference between our approach and that used in [22] is that we do not consider the fields $Z^{A}, \Psi_{A}$ as elements in the same 3-algebra as their complex conjugates $\bar{Z}_{A}, \Psi^{A}$. (We save the bar on the spinor for the Dirac conjugate.) Rather, we deal with two vector spaces $g_{1}$ and $g_{-1}$, with bases $T^{a}$ and $T_{a}$, respectively. These two vector spaces generate a graded Lie algebra $g$. We do not use any metric on $g_{1}$ and $g_{-1}$ to raise and lower indices, but we use an antilinear involution $\tau$ on $g$ to go between the subspaces, $\tau\left(T^{a}\right)=T_{a}$. We also use a bilinear form on $g$ to contract upper and lower indices. We will describe this graded Lie algebra in more detail in the following section. Here we just define the components of the fields $Z^{A}, \Psi_{A}$ in $g_{1}$ to have the index structure $Z^{A}{ }_{a}, \Psi_{A a}$. The components of $\tau\left(Z^{A}\right), \tau\left(\Psi_{A}\right)$ in $g_{-1}$ are then the complex conjugates $\bar{Z}_{A}{ }^{a}, \Psi^{A a}$. That it is natural to place the indices like this can be seen from rewriting the Bagger-Lambert version of the ABJM action as follows:

$$
\begin{aligned}
\mathcal{L}=-\left(D_{\mu} Z^{A}{ }_{a}\right) & \left(D^{\mu} \bar{Z}_{A}{ }^{a}\right)-\mathrm{i} \bar{\Psi}^{A a} \gamma^{\mu} D_{\mu} \Psi_{A a} \\
& -\mathrm{i} f^{a b}{ }_{c d} \bar{\Psi}^{A d} \Psi_{A a} Z^{B}{ }_{b} \bar{Z}_{B}{ }^{c}+2 \mathrm{i} f^{a b}{ }_{c d} \bar{\Psi}^{A d} \Psi_{B a} Z^{B}{ }_{b} \bar{Z}_{A}{ }^{c} \\
& -\frac{\mathrm{i}}{2} \epsilon_{A B C D} f^{a b}{ }_{c d} \bar{\Psi}^{A c} \Psi^{B d} Z^{C}{ }_{a} Z^{D}{ }_{b}-\frac{\mathrm{i}}{2} \epsilon^{A B C D} f^{c d}{ }_{a b} \bar{\Psi}_{A c} \Psi_{B d} \bar{Z}_{C}{ }^{a} \bar{Z}_{D}{ }^{b} \\
& -V+\frac{1}{2} \epsilon^{\mu \nu \lambda}\left(f^{a b}{ }_{c d} A_{\mu}{ }^{d}{ }_{b} \partial_{\nu} A_{\lambda}{ }^{c}{ }_{a}+\frac{2}{3} f^{b d}{ }_{g c} f^{g f}{ }_{a e} A_{\mu}{ }^{a}{ }_{b} A_{\nu}{ }^{c}{ }_{d} A_{\lambda}{ }^{e}{ }_{f}\right),
\end{aligned}
$$

where the potential now takes the form

$$
\begin{aligned}
& V=\frac{2}{3} \Upsilon^{C D}{ }_{B d} \bar{\Upsilon}_{C D}{ }^{B d}, \\
& \Upsilon^{C D}{ }_{B d}=f^{a b}{ }_{c d} Z^{C}{ }_{a} Z^{D}{ }_{b} \bar{Z}_{B}{ }^{c}+f^{a b}{ }_{c d} \delta^{[C}{ }_{B} Z^{D]}{ }_{a} Z^{E}{ }_{b} \bar{Z}_{E}{ }^{c} .
\end{aligned}
$$

This action can be shown to be $\mathcal{N}=6$ supersymmetric provided that the structure constants obey

$$
f^{a[b}{ }_{d c} f^{e] d}{ }_{g h}=f^{b e}{ }_{d[g} f^{a d}{ }_{h] c}
$$

and, under complex conjugation,

$$
\left(f^{a b}{ }_{c d}\right)^{*}=f_{a b}^{c d} \equiv f_{a b}^{c d}
$$

One immediate way to see that this identity is relevant is to consider the Chern-Simons term

$$
\mathcal{L}_{\mathrm{CS}}=\frac{1}{2} \varepsilon^{\mu \nu \lambda}\left(A_{\mu}{ }^{b}{ }_{a} \partial_{\nu} \tilde{A}_{\lambda}{ }^{a}{ }_{b}+\frac{2}{3} A_{\mu}{ }^{a}{ }_{b} \tilde{A}_{\nu}{ }^{b}{ }_{c} \tilde{A}_{\lambda}{ }^{c} a\right)
$$

where we use vector fields $A_{\mu}{ }^{a} b$ and

$$
\tilde{A}_{\mu}{ }^{a} b=f^{a c}{ }_{b d} A_{\mu}{ }^{d}{ }^{c} \text {. }
$$

Identity (3.5) then follows from the observation that when deriving the field equation the variation of each vector field must provide an identical contribution to the answer. Note that also the Chern-Simons field without tilde has an upper and a lower index which is not the case in previous treatments of the M2-brane system.

As we will see in the following section, the structure constants can also be written as $f_{a}{ }^{b}{ }_{c}{ }^{d}$. It is then interesting to note that they, as well as their corresponding fundamental 
identity, appear naturally also in the embedding tensor formalism of [27] but for seemingly completely different reasons.

In terms of structure constants of generalized Jordan triple systems the transformation rules for the six supersymmetries, parametrized by the complex self-dual three-dimensional spinor $\epsilon_{A B}$, read

$\delta Z^{A}{ }_{a}=\mathrm{i} \bar{\epsilon}^{A B} \Psi_{B a}$,

$\delta \Psi_{B d}=\gamma^{\mu} D_{\mu} Z^{A}{ }_{d} \epsilon_{A B}+f^{a b}{ }_{c d} Z^{C}{ }_{a} Z^{D}{ }_{b} \bar{Z}_{B}{ }^{c} \epsilon_{C D}-f^{a b}{ }_{c d} Z^{A}{ }_{a} Z^{C}{ }_{b} \bar{Z}_{C}{ }^{c} \epsilon_{A B}$,

while the Chern-Simons 1-form transforms as follows:

$$
\delta A_{\mu}{ }^{a}{ }_{b}=-\mathrm{i} \bar{\epsilon}_{A B} \gamma_{\mu} \Psi^{A a} Z_{b}^{B}+\mathrm{i} \bar{\epsilon}^{A B} \gamma_{\mu} \Psi_{A b} \bar{Z}_{B}{ }^{a} .
$$

To prove that the Lagrangian has six supersymmetries only requires the use of identities (3.5) and (3.6). The latter is needed since $\tau$ is antilinear. We have for example

$$
\tau\left(f^{a b}{ }_{c d} Z^{A}{ }_{e}\right)=\left(f^{a b}{ }_{c d}\right)^{*} \tau\left(Z^{A}{ }_{e}\right)=f^{c d}{ }_{a b} \bar{Z}_{A}{ }^{e} .
$$

This also ensures that the kinetic term in the Lagrangian is positive definite. In order to see how identity (3.5) arises in generalized Jordan triple systems, we need to discuss some further aspects of the underlying graded Lie algebra.

\section{Triple systems and graded Lie algebras}

In this section we will describe how the two vector spaces $g_{1}$ and $g_{-1}$, with bases $T^{a}$ and $T_{a}$, respectively, generate a graded Lie algebra $g$. The fact that $g$ is graded means that $g$ can be written as a direct sum of subspaces $g_{k}$ for all integers $k$, such that

$$
\left[g_{i}, g_{j}\right] \subseteq g_{i+j}
$$

for all integers $i, j$ (with the possibility that $g_{k}=0$ for all sufficiently large $|k|$ ). We call $k$ the level of the elements in $g_{k}$.

It follows in particular that any subspace $g_{k}$ form a representation of the subalgebra $g_{0}$. First we consider as $g_{0}$ the Lie algebra $s l(n)$, with generators $K^{a}{ }_{b}$ and commutation relations

$$
\left[K_{b}^{a}, K_{d}^{c}\right]=\delta_{b}^{c} K_{d}^{a}-\delta_{d}^{a} K_{b}^{c} .
$$

We let $s l(n)$ act on $g_{1}$ and $g_{-1}$ in the fundamental and antifundamental representations, respectively:

$$
\left[K^{a}{ }_{b}, T^{c}\right]=\delta_{b}{ }^{c} T^{a}, \quad\left[K^{a}{ }_{b}, T_{c}\right]=-\delta^{a}{ }_{c} T_{b} .
$$

In the graded Lie algebra we must also have $\left[g_{-1}, g_{1}\right] \subseteq g_{0}$. For this we introduce the structure constants $f^{a}{ }_{b}{ }^{c}$ by

$$
\left[T^{a}, T_{b}\right]=f_{b{ }_{b}{ }^{c}} K^{d}{ }_{c} \equiv S_{b}^{a},
$$

and from (4.3) we get

$$
\left[S^{a}{ }_{b}, T^{c}\right]=f_{b}^{a}{ }_{b} T^{d}, \quad\left[S^{a}{ }_{b}, T_{c}\right]=-f_{b}^{a}{ }_{c}{ }_{c} T_{d} .
$$

We thus have $\left[\left[T^{a}, T_{b}\right], T^{c}\right]=f^{a}{ }_{b}{ }^{c}{ }_{d} T^{d}$, and analogously we define the structure constants $f_{a}{ }^{b}{ }_{c}{ }^{d}$ by $\left[\left[T_{a}, T^{b}\right], T_{c}\right]=f_{a}{ }^{b}{ }^{d} T_{d}$. It follows from (4.5) that

$$
f_{a}{ }^{b}{ }^{d}=f^{b}{ }_{a}{ }^{d} \text {. }
$$

For the Jacobi identity

$$
\left[\left[T^{a}, T_{b}\right], T^{c}\right]-\left[\left[T^{c}, T_{b}\right], T^{a}\right]=\left[\left[T^{a}, T^{c}\right], T_{b}\right]
$$


to hold, the structure constants must satisfy the identity

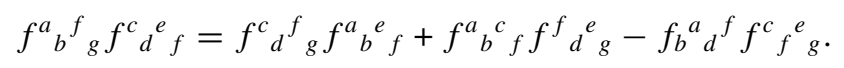

We can now redefine $g_{0}$ to be the subalgebra of $s l(n)$ spanned by all elements $S^{a}{ }_{b}$ (so that $\left.\left[g_{1}, g_{-1}\right]=g_{0}\right)$ with the commutation relations

$$
\left[S_{b}^{a}, S_{d}^{c}\right]=f_{b}^{a}{ }_{e}^{c} S_{d}^{e}-f_{b}{ }^{a}{ }^{e} S^{c}{ }_{e} .
$$

Let $\tau$ be the restriction of the Chevalley involution on $s l(n)$ to $g_{0}$. Then $\tau$ can be extended by $\tau\left(T^{a}\right)=T_{a}$ to a graded involution on the whole of $g$, such that $\tau\left(g_{k}\right)=g_{-k}$ for all integers $k$. It follows from this property, together with (4.1), that $g_{1}$ closes under the triple product

$$
(a b c)=[[a, \tau(b)], c],
$$

(and likewise for $g_{-1}$ ). Thus identity (4.8) can be expressed as

$$
(a b(x y z))-(x y(a b z))=((a b x) y z)-(x(b a y) z) .
$$

This is the definition of a generalized Jordan triple system, a vector space with a triple product that satisfies (4.11). Thus any graded Lie algebra with a graded involution leads to a generalized Jordan triple system. Conversely, for any generalized Jordan triple system $T$, there is an associated graded Lie algebra $g$, which is an extension of the vector space $g_{-1}+g_{0}+g_{1}$ that we described above [28-31].

We stress that the Lie algebra associated with a generalized Jordan triple system is the whole graded Lie algebra $g$, and not only the subalgebra $g_{0}$, which (in the case of 3-algebras) was called the associated Lie algebra by Bagger and Lambert in [3]. The graded Lie algebra $g$ associated with a generalized Jordan triple system $T$ was constructed by Kantor in a way such that if $g$ is finite dimensional, then simplicity of $g$ is equivalent to $K$-simplicity of $T$ [28, 29]. A generalized Jordan triple system $T$ is $K$-simple if there is no proper non-trivial subspace $U$ such that $(T T U) \subseteq U$ and $(U T T) \subseteq U$.

In the construction of the graded Lie algebra associated with a generalized Jordan triple system, one defines generators $T^{a b}=\left[T^{a}, T^{b}\right]$ at level two, $T^{a b c}=\left[\left[T^{a}, T^{b}\right], T^{c}\right]$ at level three, and so on (and likewise $T_{a b}, T_{a b c}, \ldots$ at the negative levels). These elements will satisfy

$$
0=T^{(a b) c d \cdots}=T^{[a b c] d \cdots},
$$

due to antisymmetry of the Lie bracket and the Jacobi identity, but also further conditions that amount to factoring out ideals from the free Lie algebra generated by $T^{a}$ and $T_{a}$.

Assume that $g_{0}$ is semisimple. We can extend the Killing form $\kappa$ on $g_{0}$ to the vector space $g_{-1}+g_{0}+g_{1}$ by $\kappa\left(T^{a}, T_{b}\right)=\delta^{a}{ }_{b}$. Then we can recursively (using the invariance) extend it to an invariant bilinear form on the whole of the free Lie algebra generated by $T^{a}$ and $T_{a}$, provided that the structure constants satisfy

$$
f_{b{ }^{c}{ }^{c}}=f^{c}{ }_{d}^{a} b \text {. }
$$

But this invariant bilinear form will then be degenerated, and the corresponding ideals are exactly those that we have to factor out to obtain the Lie algebra associated with the generalized Jordan triple system. This can be done recursively. Suppose that the restriction of the bilinear form to the vector space $g_{-k+1}+\cdots+g_{k-1}$, for some $k$, is non-degenerate. Then $\kappa(x, y)$, where $x \in g_{k}$ and $y \in g_{-k}$, is a linear combination of terms

$f^{a_{1} \cdots a_{k}}{ }_{b_{1} \cdots b_{k}} \equiv(-1)^{k+1} \kappa\left(T^{a_{1} \cdots a_{k}}, T_{b_{k} \cdots b_{1}}\right)=(-1)^{k} \kappa\left(\left[T^{a_{1} \cdots a_{k}}, T_{b_{1}}\right], T_{b_{k} \cdots b_{2}}\right)$.

Using the structure constants for the triple product, this can be evaluated as

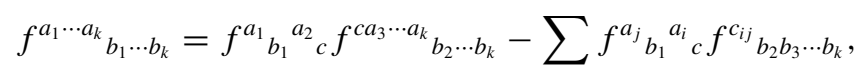

6 
where the sum goes over all $i, j$ such that $1 \leqslant i<j \leqslant k$ and $c_{i j}$ denotes the sequence of indices obtained from $a_{1} \cdots a_{n}$ by omitting $a_{j}$ and replacing $a_{i}$ by $c$, that is,

$$
c_{i j}=a_{1} \cdots a_{i-1} c a_{i+1} \cdots a_{j-1} a_{j+1} \cdots a_{k} .
$$

From now on, we assume that the structure constants are antisymmetric in the first and third indices. It then follows from (4.13) that they are antisymmetric also in the second and fourth indices:

$$
f_{b{ }^{c}{ }^{c}}^{a}=-f_{b{ }_{b}{ }^{a}}=-f^{a}{ }_{d b}^{c}
$$

and we have

$$
f^{a b}{ }_{c d}=2 f_{c b}^{a b}
$$

from the Jacobi identity. Identity (4.8) then becomes

$$
f^{e[a}{ }_{d c} f^{b] d}{ }_{g h}=f_{d[g}^{a b} f_{h] c}^{e d},
$$

which is precisely the identity needed in the previous section to prove supersymmetry. Furthermore, we have

$$
\begin{aligned}
\left(f^{a}{ }_{b d}{ }^{c}\right)^{*} & =\kappa\left(\tau\left(f^{a}{ }_{b}{ }^{c}{ } T^{e}\right), T^{d}\right)=\kappa\left(\tau\left(\left[\left[T^{a}, T_{b}\right], T^{c}\right]\right), T^{d}\right) \\
& =\kappa\left(\left[\left[T_{a}, T^{b}\right], T_{c}\right], T^{d}\right)=f_{a}{ }^{b}{ }^{d}{ }^{d}=f^{b}{ }_{a}{ }^{d}{ }_{c}
\end{aligned}
$$

since $\tau$ is antilinear, and using (4.18) we get $\left(f_{c d}^{a b}\right)^{*}=f^{c d}{ }_{a b}$. Thus the requirements for the six supersymmetries of the action (3.2) are satisfied.

With the antisymmetry $f^{a}{ }_{b}{ }^{c}{ }=-f^{c}{ }^{a}{ }^{a}{ }$, the first term on the right-hand side of (4.15) coincide with the first term in the summation. In the case $k=3$ the equation simplifies to

$$
\begin{aligned}
f^{a b c}{ }_{d e f} & =2 f^{a}{ }_{d}{ }_{g} f^{g c}{ }_{e f}-f^{c}{ }_{d}{ }_{g} f^{g b}{ }_{e f}-f^{c}{ }_{d}{ }_{g} f^{a g}{ }_{e f} \\
& =f^{a b}{ }_{d g} f^{g c}{ }_{e f}-\frac{1}{2} f^{c a}{ }_{d g} f^{g b}{ }_{e f}-\frac{1}{2} f^{c b}{ }_{d g} f^{a g}{ }_{e f} \\
& =f^{a b}{ }_{d g} f^{g c}{ }_{e f}-f^{c[a}{ }_{g d} f^{b] g}{ }_{e f} .
\end{aligned}
$$

We see that $f^{a b c}$ def is antisymmetric in the first two indices and vanishes upon antisymmetrization in the three upper indices (or the three lower ones). This is in accordance with the Jacobi identity, since by definition

$$
f_{d e f}^{a b c}=\kappa\left(\left[\left[T^{a}, T^{b}\right], T^{c}\right],\left[\left[T_{f}, T_{e}\right], T_{d}\right]\right) .
$$

Continuing in this way, one can determine which symmetries the tensors at each level must have, and their commutation relations follow from the Jacobi identity. Thus the graded Lie algebra $g$ is completely determined by the generalized Jordan triple system $T$, or equivalently, by the structure constants $f^{a}{ }_{b}{ }^{c} d$.

We will now discuss some further properties of the Lie algebra $g$. We will make use of the following two theorems by Kantor, the first of which we mentioned already in the beginning of this section.

Theorem 1 [29] (section 3, prop. $7^{\prime}$ and theorem 1', see also [30] theorem 3.5). Assume that $g$ is finite dimensional. Then $g$ is simple if and only if $T$ is $K$-simple.

Theorem 2 [29] (section 4, prop. 12). Assume that $g$ is finite dimensional and simple. Then there are nonzero elements $e, f, h$, at level one, minus one and zero, respectively, that satisfy the Chevalley relations

$$
[h, e]=2 e, \quad[h, f]=-2 f, \quad[e, f]=h .
$$

Thus $e, f, h$ are the Chevalley basis elements corresponding to a simple root. Theorems 1 and 2 together give the following corollary. 
Corollary 3. If $T$ is $K$-simple and antisymmetric in the first and third arguments, then $g$ is infinite dimensional.

Proof. Suppose the contrary, that $g$ is finite dimensional. It follows by theorem 1 that $g$ is simple. But then according to theorem 2 there are nonzero elements $e, f, h$ such that

$$
[[e, f], e]=[h, e]=2 e .
$$

On the other hand, we have

$$
[[e, f], e]=(e \tau(f) e)=0
$$

since the triple product is antisymmetric in its first and third arguments. Thus we get a contradiction and we conclude that $g$ is infinite dimensional.

In particular, for the totally antisymmetric triple system used in the BLG theory with eight supersymmetries, the associated Lie algebra $g$ is infinite dimensional. Indeed, this triple system is $K$-simple since the structure constants are proportional to the $s o(4)$ epsilon tensor. We stress that although $g$ is infinite dimensional, each of the infinitely many subspaces $g_{k}$ is finite dimensional. Again, $g$ should not be confused with its subalgebra $g_{0}$ (which is $s o(4)$ in this case), nor with the triple system itself (which can be identified with $g_{1}$ ).

There is still the possibility that $g$ is an infinite-dimensional Kac-Moody algebra. From theorem 2 we only know that in the finite-dimensional case, it is possible to find a Chevalley basis and a simple root such that the corresponding elements $e, f, h$ belong to level one, minus one and zero, respectively. If $g$ is an infinite-dimensional Kac-Moody algebra then the grading cannot be given by a simple root in this way. It might be possible to find elements $e, f, h$ at level one, minus one and zero, respectively, such that $e$ and $f$ are eigenvectors to the adjoint action of $h=[e, f]$. But then the eigenvalues must be zero instead of \pm 2 . This suggests that $g$ is Borcherds algebra [32], or some even more general algebra that (unlike a Kac-Moody algebra) allow for such zero eigenvalues.

We are finally able to express the ABJM action completely in terms of the associated graded Lie algebra $g$. We recall that $Z^{A}, \Psi_{A}$ are elements in $g_{1}$, while $Z_{A}, \Psi^{A}$ are elements in $g_{1}$, which are mapped onto $Z^{A}, \Psi_{A}$ under the involution,

$$
\begin{array}{ll}
Z^{A}=Z_{a}^{A} T^{a}, & Z_{A}=Z_{A}{ }^{a} T_{a}, \\
\Psi_{A}=\Psi_{A a} T^{a}, & \Psi^{A}=\Psi^{A a} T_{a},
\end{array}
$$

and $A_{\mu}$ belongs to the $g_{0}$ subalgebra:

$$
A_{\mu}=A_{\mu}^{a}{ }_{b}^{b}{ }_{a} \text {. }
$$

The Lagrangian (3.2) can thus be rewritten as

$$
\begin{aligned}
\mathcal{L}=-\kappa\left(D^{\mu} \bar{Z}_{A}, D_{\mu} Z^{A}\right)-\mathrm{i} \kappa\left(\bar{\Psi}^{A}, \gamma^{\mu} D_{\mu} \Psi_{A}\right) \\
+\mathrm{i} \kappa\left(\left[\bar{\Psi}^{A}, \bar{Z}_{B}\right],\left[\Psi_{A}, Z^{B}\right]\right)-2 \mathrm{i} \kappa\left(\left[\bar{\Psi}^{A}, \bar{Z}_{A}\right],\left[\Psi_{B}, Z^{B}\right]\right) \\
-\frac{\mathrm{i}}{2} \epsilon_{A B C D} \kappa\left(\left[\bar{\Psi}^{A}, \Psi^{B}\right],\left[Z^{C}, Z^{D}\right]\right)-\frac{\mathrm{i}}{2} \epsilon^{A B C D} \kappa\left(\left[\bar{Z}_{A}, \bar{Z}_{B}\right],\left[\bar{\Psi}_{C}, \Psi_{D}\right]\right) \\
\\
-V+\epsilon^{\mu \nu \lambda}\left(\kappa\left(\partial_{\mu} A_{\nu}, A_{\lambda}\right)-\frac{2}{3} \kappa\left(\left[A_{\mu}, A_{\nu}\right], A_{\lambda}\right)\right),
\end{aligned}
$$

where, after using (4.21), the potential takes the simple form

$$
V=\kappa\left(\left[\left[\bar{Z}_{A}, \bar{Z}_{B}\right], Z^{C}\right],\left[\left[Z^{A}, Z^{B}\right], \bar{Z}_{C}\right]\right)-\frac{1}{3} \kappa\left(\left[\left[\bar{Z}_{A}, \bar{Z}_{B}\right], \bar{Z}_{C}\right],\left[\left[Z^{A}, Z^{B}\right], Z^{C}\right]\right) .
$$


One natural generalization would be to let $Z^{A}$ and $\psi_{A}$ take values in $g_{k}$ for all positive levels $k$, instead of just $g_{1}$. This does not, however, seem to be compatible with supersymmetry.

\section{Conclusions and comments}

This paper is based on the observation that the $\mathcal{N}=6 \mathrm{ABJ}$ theory can be written in terms of four-index structure constants $f^{a c} b d$ which are antisymmetric only in the upper pair and the lower pair separately. The fundamental identity then takes the same form as the basic identity in a generalized Jordan triple system suggesting a connection to graded Lie algebras associated with such triple systems. To rewrite the theory, we use an involution and an invariant bilinear form on the Lie algebra, which naturally induce a metric on the generalized Jordan triple system. However, this means that we do not need to use the metric explicitly in constructing the Lagrangian.

We have been very general in the description of the Lie algebra associated with a generalized Jordan triple system. The example that it first of all should be applied to is the 3-algebra given by Bagger and Lambert in [22]. The relation between their work and ours should be studied in detail. Also, the position of the indices suggests an interesting connection to the embedding tensor method used in [27].

Even if much of what we have presented in this paper are based on reformulations of previous results, we think that our approach opens up new perspectives. We have interpreted the fields $Z^{A}, \Psi_{A}$ as elements in $g_{1}$, their conjugates as elements in $g_{-1}$, and the gauge field $A_{\mu}$ as an element in $g_{0}$. Although we do not have any interpretation of the elements at higher (positive and negative) levels, we cannot set them to zero, because we need the triple product to be antisymmetric in the first and third arguments. Therefore we believe that also the full algebra might play an important role in the theory of M2-branes. For example, it points out a new direction in which one could possibly search for the behavior $n^{3 / 2}$ that the degrees of freedom of $n$ M2-branes are conjectured to exhibit. In any case, it would be interesting to see how fast the dimension of the Lie algebra grows as we go to higher levels. The algorithm that we have described for finding the corresponding $g_{0}$-representations would probably be easy to implement in a computer program.

There are many implications following from a relation between M2-brane systems and generalized Jordan triple systems. In particular, very little is known about the structure of such triple systems when the grading is infinite. Finite-dimensional cases are better known and many of their properties have been studied (for an overview of Jordan, Kantor and Freudenthal triple systems, we refer to [33]). For instance, in analogy with Freudenthal triple systems (see e.g. [34]), we may suspect that the generalized Jordan triple systems used here might also be of interest in connection with minimal representations, spherical vectors and the associated automorphic forms. For previous attempts to use the theory of automorphic forms in the context of the M2-brane, see [35, 36].

Let us end by mentioning two other issues. The Freudenthal triple system construction leads to minimal representations via nonlinear realizations of the full algebra [34]. In [18] the authors argue that the M2-theory discussed here really has eight supersymmetries but that the last two are somehow realized non-locally. The connection to triple systems may in fact suggest how to derive nonlinear realizations also of the remaining two supersymmetries needed to obtain the maximal number of $\mathcal{N}=8$ supersymmetries.

The second issue is that of unitarity. Standard triple system constructions naturally lead to Lie algebras that appear in their split form although other forms are also possible. To achieve unitarity one may try to quantize the theory whereby an infinite-dimensional unitary minimal representation is realized on a Hilbert space. For an explicit example, see [37]. 


\section{Acknowledgments}

We would like to thank Joakim Arnlind, Ling Bao, Ulf Gran, Andreas Gustavsson, Carlo Meneghelli, Christoffer Petersson and Hidehiko Shimada for discussions. The work is partly funded by the Swedish Research Council.

\section{References}

[1] Bagger J and Lambert N 2007 Modeling multiple M2's Phys. Rev. D 75045020 (arXiv:hep-th/0611108)

[2] Gustavsson A 2007 Algebraic structures on parallel M2-branes arXiv:0709.1260 [hep-th]

[3] Bagger J and Lambert N 2008 Gauge symmetry and supersymmetry of multiple M2-branes Phys. Rev. D 77065008 (arXiv:0711.0955 [hep-th])

[4] Bagger J and Lambert N 2008 Comments on multiple M2-branes J. High Energy Phys. JHEP02(2008)105 (arXiv:0712.3738 [hep-th])

[5] Schwarz J H 2004 Superconformal Chern-Simons theories J. High Energy Phys. JHEP11(2004)078 (arXiv:hep-th/0411077)

[6] Papadopoulos G 2008 M2-branes, 3-Lie algebras and Plücker relations J. High Energy Phys. JHEP05(2008)054 (arXiv:0804.2662 [hep-th])

[7] Gauntlett J P and Gutowski J B 2008 Constraining maximally supersymmetric membrane actions arXiv:0804.3078 [hep-th]

[8] Lambert N and Tong D 2008 Membranes on an orbifold arXiv:0804.1114 [hep-th]

[9] Distler J, Mukhi S, Papageorgakis C and Van Raamsdonk M 2008 M2-branes on M-folds arXiv:0804.1256 [hep-th]

[10] Gran U, Nilsson B E W and Petersson C 2008 On relating multiple M2 and D2-branes arXiv:0804.1784 [hep-th]

[11] Gomis J, Milanesi G and Russo J G 2008 Bagger-Lambert theory for general Lie algebras J. High Energy Phys. JHEP06(2008)075 (arXiv:0805.1012 [hep-th])

[12] Benvenuti S, Rodriguez-Gomez D, Tonni E and Verlinde H $2008 \mathrm{~N}=8$ superconformal gauge theories and M2 branes arXiv:0805.1087 [hep-th]

[13] Ho P M, Imamura Y and Matsuo Y 2008 M2 to D2 revisited J. High Energy Phys. JHEP07(2008)003 (arXiv:0805.1202 [hep-th])

[14] Bandres M A, Lipstein A E and Schwarz J H 2008 Ghost-free superconformal action for multiple M2-branes arXiv:0806.0054 [hep-th]

[15] Gomis J, Rodriguez-Gomez D, Van Raamsdonk M and Verlinde H 2008 Supersymmetric Yang-Mills theory from Lorentzian three-algebras arXiv:0806.0738 [hep-th]

[16] Ezhuthachan B, Mukhi S and Papageorgakis C 2008 D2 to D2 J. High Energy Phys. JHEP07(2008)041 (arXiv:0806.1639 [hep-th])

[17] Cecotti S and Sen A 2008 Coulomb branch of the Lorentzian three algebra theory arXiv:0806.1990 [hep-th]

[18] Aharony O, Bergman O, Jafferis D L and Maldacena J $2008 N=6$ superconformal Chern-Simons-matter theories, M2-branes and their gravity duals arXiv:0806.1218 [hep-th]

[19] Van Raamsdonk M 2008 Comments on the Bagger-Lambert theory and multiple M2-branes arXiv:0803.3803 [hep-th]

[20] Benna M, Klebanov I, Klose T and Smedback M 2008 Superconformal Chern-Simons theories and $\mathrm{AdS}_{4} / \mathrm{CFT}_{3}$ correspondence arXiv:0806.1519 [hep-th]

[21] Bandres M A, Lipstein A E and Schwarz J H 2008 Studies of the ABJM theory in a formulation with manifest $S U$ (4) $R$-symmetry arXiv:0807.0880 [hep-th]

[22] Bagger $\mathrm{J}$ and Lambert $\mathrm{N} 2008$ Three-algebras and $N=6$ Chern-Simons gauge theories arXiv:0807.0163 [hep-th]

[23] Gaiotto D and Witten E 2008 Janus configurations, Chern-Simons couplings, and the theta-angle in $N=4$ super Yang-Mills theory arXiv:0804.2907 [hep-th]

[24] Hosomichi K, Lee K M, Lee S, Lee S and Park J $2008 N=4$ Superconformal Chern-Simons theories with hyper and twisted hyper multiplets J. High Energy Phys. JHEP07(2008)091 (arXiv:0805.3662 [hep-th])

[25] Hosomichi K, Lee K M, Lee S, Lee S and Park J $2008 N=5$, 6 Superconformal Chern-Simons theories and M2-branes on orbifolds J. High Energy Phys. JHEP09(2008)002 (arXiv:0806.4977 [hep-th])

[26] Schnabl M and Tachikawa Y 2008 Classification of $N=6$ superconformal theories of ABJM type arXiv:0807.1102 [hep-th]

[27] Bergshoeff E A, Hohm O, Roest D, Samtleben H and Sezgin E 2008 The superconformal gaugings in three dimensions arXiv:0807.2841 [hep-th] 
[28] Kantor I 1970 Graded Lie algebras Trudy Sem. Vekt. Tenz. Anal. 15 227-66

[29] Kantor I L 1972 Some generalizations of Jordan algebras Trudy Sem. Vekt. Tenz. Anal. 16 407-99

[30] Asano H and Kaneyuki S 1988 Graded Lie algebras and generalized Jordan triple systems Nagoya Math. J. 112 $81-115$

[31] Palmkvist J 2007 Generalized conformal realizations of Kac-Moody algebras arXiv:0711.0441 [hep-th]

[32] Borcherds R E 1988 Generalized Kac-Moody algebras J. Algebra 115501

[33] Palmkvist J 2006 A realization of the Lie algebra associated to a Kantor triple system J. Math. Phys. 47023505 (arXiv:math/0504544)

[34] Gunaydin M, Koepsell K and Nicolai H 2001 Conformal and quasiconformal realizations of exceptional Lie groups Commun. Math. Phys. 22157 (arXiv:hep-th/0008063)

[35] Pioline B, Nicolai H, Plefka J and Waldron A $2001 R^{4}$ couplings, the fundamental membrane and exceptional theta correspondences J. High Energy Phys. JHEP03(2001)036 (arXiv:hep-th/0102123)

[36] Pioline B and Waldron A 2004 The automorphic membrane J. High Energy Phys. JHEP06(2004)009 (arXiv:hep-th/0404018)

[37] Gunaydin M, Koepsell K and Nicolai H 2002 The minimal unitary representation of E8(8) Adv. Theor. Math. Phys. 5923 (arXiv:hep-th/0109005) 